\title{
Double-Peaked Profiles: Ubiquitous Signatures of Disks in the Broad Emission Lines of Active Galactic Nuclei
}

\author{
T. Storchi-Bergmann ${ }^{1,4}$, J. S. Schimoia ${ }^{1,3}$, B. M. Peterson ${ }^{2,3}$, M. Elvis ${ }^{4}$, K. D. Denney ${ }^{3}$, M. Eracleous ${ }^{5}$, and R. S. Nemmen ${ }^{6}$ \\ ${ }^{1}$ Instituto de Física, Universidade Federal do Rio Grande do Sul, Campus do Vale, Porto Alegre, RS, Brazil; thaisa@ufrgs.br \\ ${ }^{2}$ Space Telescope Science Institute, 3700 San Martin Drive, Baltimore, MD 21218, USA \\ ${ }^{3}$ Department of Astronomy, 140 West 18th Avenue, and the Center for Cosmology and AstroParticle Physics, \\ 191 West Woodruff Avenue, The Ohio State University, Columbus, OH 43210, USA \\ ${ }^{4}$ Harvard-Smithsonian Center for Astrophysics, 60 Garden Street, Cambridge, MA 02138, USA \\ ${ }^{5}$ Department of Astronomy \& Astrophysics, The Pennsylvania State University, University Park, PA 16802, USA \\ ${ }^{6}$ Instituto de Astronomia, Geofísica e Ciências Atmosféricas, Universidade de São Paulo, 05508-090 São Paulo, SP, Brazil \\ Received 2016 February 4; revised 2016 November 17; accepted 2016 December 9; published 2017 January 31
}

\begin{abstract}
Broad $\left(\sim 10,000 \mathrm{~km} \mathrm{~s}^{-1}\right)$, double-peaked emission-line profiles of Balmer lines emitted by active galactic nuclei (AGN) are thought to originate in the outer parts of an accretion disk surrounding a nuclear supermassive black hole $(\mathrm{SMBH})$, at $\sim 1000$ gravitational radii, and are most frequently observed in the nuclear spectra of low-luminosity AGN (LLAGN) and radio galaxies. In the present paper we argue that broad double-peaked profiles are present also in the spectra of other type 1 AGN, such as Seyfert 1 galaxies, suggesting that the inner part of the broad-line region (BLR) is also the outer part of the accretion disk. We use the Palomar spectral survey of nearby galaxies to show that the only difference between Seyfert 1 BLR line profiles and "bona fide" double-peakers is that, in most cases, besides a disk component, we need an additional Gaussian component attributed to nondisk clouds. The recognition that the inner and most variable part of the BLR has a disk geometry suggests that the factor $f$ in the expression to obtain the SMBH mass in type 1 AGN, $M_{\mathrm{BH}}=f\left(R_{\mathrm{BLR}} \Delta V^{2} / G\right)$, is $f=1 / \sin ^{2} i$ for the disk-dominated sources. Our median $i=27^{\circ}$ implies $f=4.5$, very close to the most recent value of $f=4.3 \pm 1.05$, obtained from independent studies. We derive a relation between $f$ and the FWHM of the broad profile that may help to reduce the uncertainties in the SMBH mass determinations of AGN.
\end{abstract}

Key words: accretion, accretion disks - galaxies: active - galaxies: nuclei - galaxies: Seyfert - line: profiles

\section{Introduction}

Double-peaked emission lines in the spectra of active galactic nuclei (AGN), usually observed in the permitted $\mathrm{H}$ and He lines, are believed to originate in the outer parts of a disk, typically at about 1000 gravitational radii $\left(R_{g}=G M_{\mathrm{BH}} / c^{2}\right)$ from the supermassive black hole (SMBH) of mass $M_{\mathrm{BH}}$. This line-emitting region is often taken to be the outer regions of the accretion disk that extends down to a few $R_{g}$. A disk origin for double-peaked emission lines was first proposed by Chen et al. (1989) and Chen \& Halpern (1989) for the archetypical "double-peaker" Arp 102B. Subsequent work by Eracleous \& Halpern $(1994,2003)$ found a number of other sources, confirming that a disk (or ring) model does reproduce the double-peaked profiles. A similar conclusion was reached by Strateva et al. (2003) for a sample of 116 AGN with doublepeaked Balmer line profiles selected from the Sloan Digital Sky Survey.

There have been other suggestions for the origin of doublepeaked emission-line profiles. Gaskell (1983) and Peterson et al. (1987) suggested that they might be the signature of binary black holes. Zheng et al. (1990) pointed out that a doube-peaked profile could originate in a biconical outflow, while Wanders et al. (1995) and Goad \& Wanders (1996) showed that it could be produced by an anisotropic continuum source, or, for that matter, a highly anisotropic distribution of emission-line gas. The preponderance of evidence, however, still points to a moderately to highly inclined disk-like structure (Eracleous \& Halpern 2003).
Some double-peaked profiles have been monitored spectroscopically over timescales of years, at typical intervals of months. Examples are the cases of 3C 390.3 (Veilleux \& Zheng 1991; Sergeev et al. 2002, 2011; Jovanovic et al. 2010; Shapovalova et al. 2010; Popovic et al. 2011), which has also been monitored on reverberation timescales (Dietrich et al. 1998, 2012), NGC 1097 (Storchi-Bergmann et al. 2003), Arp 102B (Sergeev et al. 2000; Shapovalova et al. 2013), and 14 other double-peaked emitters (Gezari et al. 2007; Lewis et al. 2010). These observations have revealed that the doublepeaked Balmer line profiles in these sources display variability on timescales ranging from months to years. The changes in the profiles are typically varying asymmetries in the blue and/or red peaks of the profiles on the dynamical timescale, associated with the rotation of the gas or patterns in the gas surrounding the SMBH. In the particular case of NGC 1097, during the 30 $\mathrm{yr}$ it has been spectroscopically monitored (Storchi-Bergmann et al. 2003; Schimoia et al. 2012), the relative intensity of the blue and red peaks has alternated between a stronger blue peak and a stronger red peak on timescales of months. This behavior has been attributed to a spiral arm rotating in the disk, with a rotation period of about $\sim 18$ months.

Most of the monitoring campaigns of double-peakers reported to date were carried out using very sparse cadences, typically one observation per month or even sparser. But recent monitoring of the nucleus of NGC 1097 by Schimoia et al. (2012) included a few observations at weekly intervals and showed that there are variations in the total flux of the line and in the separation between the blue and red peaks on a timescale 
of a week, i.e., on the reverberation timescale. In order to search for possible shorter-timescale variations, and to try to identify the driving source of the line variations, we subsequently monitored the double-peaked $\mathrm{H} \alpha$ profile in the nuclear spectrum of NGC 1097 with the SOAR telescope and the Swift satellite (Schimoia et al. 2015). These observations confirmed that the integrated flux of the line and the velocity separation between the blue and red peaks vary on a timescale as short as 5 days. This timescale is compatible with the lightcrossing time between the center of the disk and a typical radius of $1000 R_{g}$, where the emission line originates, according to our models.

The variability timescale of $\sim 5$ days, observed in NGC 1097, is of the order of the delays found between the variations of the continuum and broad emission lines in reverberation mapping (RM) studies of nearby Seyfert 1 galaxies of comparable luminosity. This indicates that the lineemitting disk in NGC 1097 is at a distance from the ionizing source that is typical of the broad-line region (BLR) of nearby Seyfert 1 galaxies (Peterson et al. 2004).

It is also interesting that many Seyfert 1 galaxies show, at least on some occasions, evidence for an underlying disk-like component. Double peaks or shoulders are frequently seen in the Balmer line profiles of highly variable and closely monitored sources like NGC 5548 (Sergeev et al. 2007). These various observations lead one to consider the possibility that the Balmer line profiles in all Seyfert 1 galaxies have a disklike component that could be identified with the outer parts of an accretion disk. A simple hypothesis might be that Balmer line profiles in Seyfert 1 galaxies are a composite of (1) a disklike component, as seen clearly in the spectra of double-peaked emitters, and (2) another component originating in clouds that are not in the disk, e.g., in a wind component or in orbits farther out.

A model of a disk component plus another narrower broad component to reproduce the broad Balmer line profiles of Seyfert 1 nuclei and quasars has been previously proposed by Popović et al. (2004) and collaborators (Bon et al. 2009; La Mura et al. 2009). A recent theoretical study by Elitzur et al. (2014) also argues that the BLR of Seyfert 1 galaxies has two parts: a disk and a system of clouds that are part of an outflowing disk wind. As the Eddington ratio drops, the high column density gas, which resides largely in the disk, dominates the emission and leads to double-peaked line profiles. A disk-wind component would be expected to be stronger at higher Eddington ratios, so this might naturally account for the relative prominence of the disk-like component in lower-luminosity $\mathrm{AGN}$ and the relative difficulty of identifying the disk component in more luminous, higher Eddington ratio objects.

In this contribution we study the spectra of all Seyfert 1 galaxies of the Palomar spectroscopic survey (Filippenko \& Sargent 1985; Ho et al. 1995)—selected for being a homogeneous and representative sample of galaxy nuclear spectra in the near universe-to show that we can identify disk-like structures in the Balmer line profiles of many of them. An underlying disk-like component can account for much of the structure and asymmetries seen in Seyfert 1 line profiles and, combined with another narrower component attributable to other emitting clouds, can reproduce most of the broad-line profiles. Thus, our primary goal is to demonstrate the plausibility of the idea that the broad Balmer line profiles of this sample of Seyfert 1 galaxies can be described well by a combination of a disk-like component and a somewhat narrower, bell-shaped component. As the Palomar sample is a carefully selected local sample, designed to find the lowestluminosity AGN, we also use our results to obtain statistics of the presence of the disk component, and we discuss the implications for the calculation of the mass of the SMBHs in type 1 AGN. This is our secondary goal.

This paper is organized as follows. In Section 2, we describe the disk model that we have used to reproduce the doublepeaked profiles of the low-luminosity AGN (LLAGN) in NGC 1097 and other sources. In Sections 3 and 4 we use this model to reproduce the double-peaked $\mathrm{H} \alpha$ profiles of LLAGN and Seyfert 1 galaxies of the Palomar survey and show the need for an additional broad Gaussian component in most cases. In Section 5, we show how the profile shape changes as a function of inclination relative to the plane of the sky. We use these results and the frequency of occurrence of the double-peaked profiles in the Palomar sample to draw inferences about the presence of disk emission in type 1 AGN. In Section 6 we discuss the implications of our findings for the determination of $\mathrm{SMBH}$ masses in type $1 \mathrm{AGN}$, and in Section 7 we present our conclusions.

\section{The Disk Model}

We use the model described by Schimoia et al. (2012), Gilbert et al. (1999), and Storchi-Bergmann et al. (2003), assuming that the broad double-peaked emission line originates in the outer parts of a relativistic Keplerian disk of gas surrounding the SMBH. The line-emitting portion of the disk is circular and located between an inner radius $\xi_{1}$ and an outer radius of $\xi_{2}$, where $\xi$ is the disk radius in units of the gravitational radius $R_{g}=G M / c^{2}$. The plane of the disk has an inclination $i$ relative to the plane of the sky (i.e., zero degrees corresponds to a disk observed face-on). Superimposed on the axisymmetric emissivity of the circular disk, there is a perturbation in the form of a spiral arm. The corresponding emissivity law is given by

$$
\begin{aligned}
\epsilon(\xi, \phi)= & \epsilon(\xi)\left\{1+\frac{A}{2} \exp \left[-\frac{4 \ln 2}{\delta^{2}}\left(\phi-\psi_{0}\right)^{2}\right]\right. \\
& \left.+\frac{A}{2} \exp \left[-\frac{4 \ln 2}{\delta^{2}}\left(2 \pi-\phi+\psi_{0}\right)^{2}\right]\right\},
\end{aligned}
$$

where

$$
\epsilon(\xi)= \begin{cases}\epsilon_{0} \xi^{-q_{1}} & , \xi_{1}<\xi<\xi_{q} \\ \epsilon_{0} \xi_{q}^{-\left(q_{1}-q_{2}\right)} \xi^{-q_{2}} & , \xi_{q}<\xi<\xi_{2}\end{cases}
$$

is the axisymmetric emissivity of the disk. The parameter $\xi_{q}$ is the radius of maximum emissivity, or saturation radius, at which the emissivity reaches a maximum and changes behavior: $q_{1}$ is the index of the emissivity law for $\xi_{1}<\xi<\xi_{q}$, and $q_{2}$ is the index for $\xi_{q}<\xi<\xi_{2} . A$ is the brightness contrast between the spiral arm and the underlying disk, and the expression between square brackets represents the decay of the emissivity of the arm as a function of the azimuthal distance $\phi-\psi_{0}$ from the ridge line to both sides of the arm, assumed to be a Gaussian function with $\mathrm{FWHM}=\delta$ (azimuthal width). 


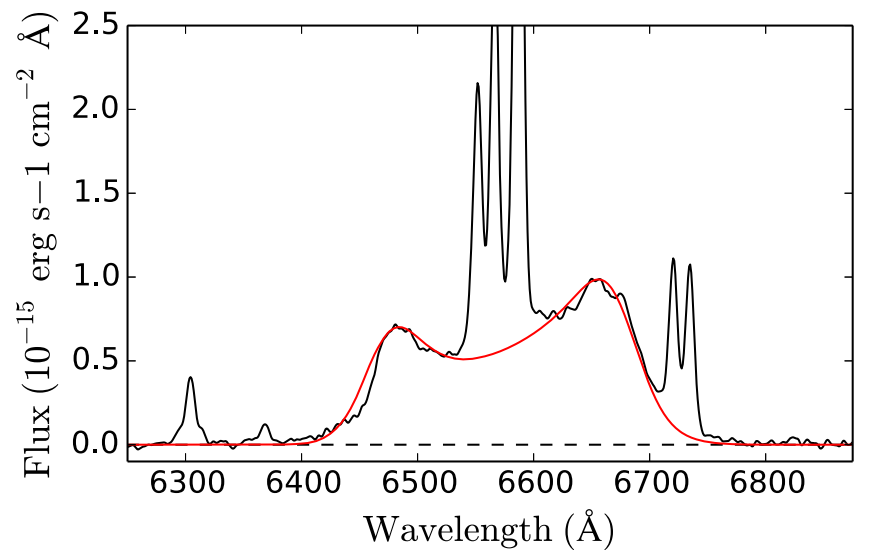

Figure 1. Fit of the accretion disk model to an $\mathrm{H} \alpha$ profile of the LLAGN in NGC 1097 (Schimoia et al. 2012). The solid black line is the observed nuclear emission spectrum, and the red solid line is the accretion disk model.

The relation between the azimuthal angle $\phi_{0}$ and the angular position $\psi_{0}$ of the ridge of emissivity of the spiral arm is given by

$$
\psi_{0}=\phi_{0}+\frac{\ln \left(\xi / \xi_{\mathrm{sp}}\right)}{\tan p},
$$

where $\phi_{0}$ is the azimuthal angle of the spiral pattern, $p$ is the pitch angle, and $\xi_{\mathrm{sp}}$ is the innermost radius of the spiral arm.

The specific intensity from each location in the disk, in the frame of the emitting particle, is calculated as

$$
I\left(\xi, \phi, \nu_{e}\right)=\frac{\epsilon(\xi, \phi)}{4 \pi} \frac{e^{-\left(\nu_{e}-\nu_{0}\right)^{2} / 2 \sigma^{2}}}{(2 \pi)^{1 / 2} \sigma},
$$

where $\nu_{e}$ is the emission frequency, $\nu_{0}$ is the rest frequency corresponding to $\mathrm{H} \alpha \lambda 6564.6$, and $\sigma$ is the local "broadening parameter" (Chen \& Halpern 1989).

The fit of this model to the $\mathrm{H} \alpha$ double-peaked profile of NGC 1097 observed on 2010 March 4 is shown in Figure 1. The model parameters are listed in Table 1. This model and its variants have been used also to reproduce varying doublepeaked profiles of five broad-line radio galaxies by Gilbert et al. (1999) and of 14 other AGN from Gezari et al. (2007) and Lewis et al. (2010).

\section{Double-Peaked Balmer Line Profiles in Nearby Seyfert 1 Galaxies}

Although previous studies-as those mentioned abovehave focused on fitting the broadest and cleanest doublepeaked profiles, many nearby Seyfert 1 nuclei also show broad Balmer lines with double peaks or shoulders. This can be observed, for example, in the nuclear spectra of Seyfert 1 galaxies, which have been the subject of spectroscopic monitoring campaigns, such as NGC 4151 (Maoz et al. 1991; Kaspi et al. 1996; Bentz et al. 2006), NGC 5548 (Netzer et al. 1990; Peterson et al. 2002; Bentz et al. 2007, 2009, 2010; Denney et al. 2010), NGC 3227 (Winge et al. 1995; Denney et al. 2010), and NGC 3516 (Denney et al. 2010). Denney et al. (2010) present the results of RM of other four Seyfert 1 nuclei besides NGC 5548 and NGC 3516. With the exception of one source, either the $\mathrm{H} \beta$ profiles are double-peaked or the residual rms spectra are double-peaked. This indicates that, even if the profile is not clearly double-peaked (e.g., due to the presence of
Table 1

Accretion Disk Parameters for H $\alpha$ Profile of NGC 1097 and $\mathrm{H} \beta \mathrm{rms}$ Profile of NGC 3227

\begin{tabular}{lcc}
\hline \hline Disk Component & NGC 1097 & $\begin{array}{c}\text { NGC 3227 } \\
\text { (rms Spectrum) }\end{array}$ \\
\hline$\xi_{1}=\xi_{\text {sp }}$ & 450 & 2000 \\
$\xi_{2}$ & 1600 & 5000 \\
$\xi_{q}$ & 1200 & 3125 \\
$i$ & 34 & 16 \\
$\phi_{0}$ & 140 & 20 \\
$q_{1}$ & -2 & -2 \\
$q_{2}$ & 3 & 3 \\
$A$ & 3.0 & 0.6 \\
$p$ & 50 & -20 \\
$\delta\left(^{\circ}\right)$ & 70 & 40 \\
$\sigma$ & 900 & 480 \\
FWHM & 10555 & 6253 \\
shift $\left(\mathrm{km} \mathrm{s}^{-1}\right)$ & $\ldots$ & -686 \\
\hline
\end{tabular}

Note. Units of $\xi_{1}, \xi_{2}$, and $\xi_{q}$ are gravitational radii.

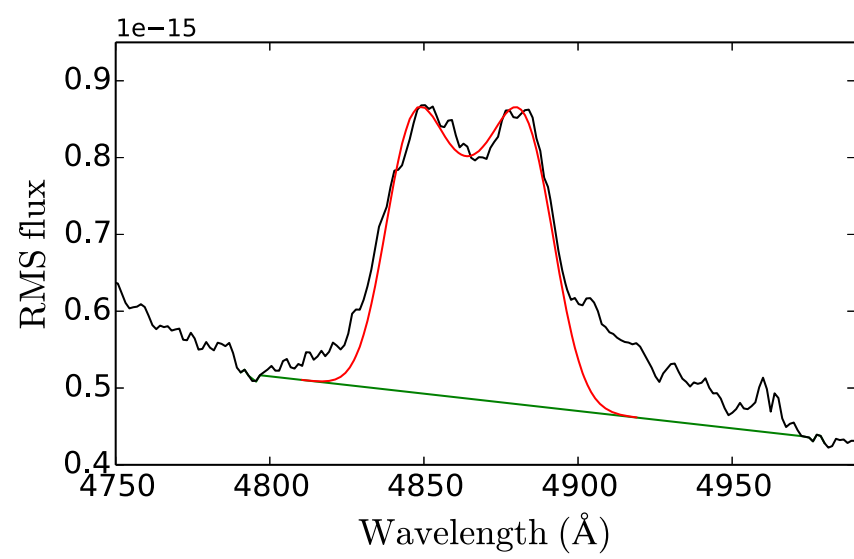

Figure 2. $\mathrm{H} \beta \mathrm{rms}$ spectrum of NGC 3227 from Denney et al. (2010); the red solid line is the fitted accretion disk model profile, while the green solid line is the adopted continuum.

other components), the most variable part of the profile is double-peaked, supporting the idea that the innermost part of the BLR (which varies most, as it is closer to the ionizing source) is a disk.

We show in Figure 2 the result of our attempt to fit the rms $\mathrm{H} \beta$ profile of NGC 3227 using our disk model and data from Denney et al. (2010): although the profiles are not always double-peaked, the rms spectrum is. The parameters of the fit are listed in Table 1. Figure 2 shows that the model does fit the double-peaked component, although there is, in addition, an even broader component, revealed by the extended red wing that could not be fitted by our model. This component probably originates at gas at higher velocities closer to the $\mathrm{SMBH}$ and/or in inflow or outflow, for example. This interpretation is consistent with the velocity-delay map of Denney et al. (2012): they conclude that there is evidence for an overall Keplerian disk-like structure, but potential evidence for an outflow (or inflow) at large velocities, particularly on the red side of the line, which appears to be at larger radii than the disk-like structure found in the main line core velocities. Five other cases 
Table 2

Nearby Type 1 AGN with Double-peaked Balmer Profiles

\begin{tabular}{|c|c|c|c|c|c|}
\hline Galaxy & Activity & $D(\mathrm{Mpc})$ & Morphology & $F_{\mathrm{dp}} / F$ & Ref. \\
\hline \multicolumn{6}{|l|}{ Palomar } \\
\hline NGC 3031 & LLAGN & 0.66 & $\mathrm{SA}(\mathrm{s}) \mathrm{ab}$ & 0.57 & A \\
\hline NGC 3227 & Sy 2 & 20.3 & SAB(s)a pec & 0.71 & B \\
\hline NGC 3516 & Sy 1.5 & 37.2 & $(\mathrm{R}) \mathrm{SB}^{0} ?(\mathrm{~s})$ & 0.91 & B \\
\hline NGC 4051 & Sy 1 & 12.7 & $\mathrm{SAB}(\mathrm{rs}) \mathrm{bc}$ & 0.74 & E \\
\hline NGC 4151 & Sy 1.5 & 17.0 & $\left(\mathrm{R}^{\prime}\right) \mathrm{SAB}(\mathrm{rs}) \mathrm{ab}$ ? & 0.63 & $\mathrm{C}$ \\
\hline NGC 4203 & LLAGN & 18.6 & $\mathrm{SAB} 0^{-} ?$ & $0.34^{*}$ & $\mathrm{D}$ \\
\hline NGC 4235 & Sy 1.2 & 37.7 & SA(s)edge-on & 0.84 & $\mathrm{E}$ \\
\hline NGC 4395 & Sy 1.8 & 8.0 & $\mathrm{SA}(\mathrm{s}) \mathrm{m}$ & 0.63 & $\mathrm{E}$ \\
\hline NGC 4450 & LLAGN & 31.1 & $\mathrm{SA}(\mathrm{s}) \mathrm{ab}$ & $0.20^{*}$ & $\mathrm{~F}$ \\
\hline NGC 4579 & Sy 2 & 25.3 & $\mathrm{SAB}(\mathrm{rs}) \mathrm{b}$ & $0.21^{*}$ & $\mathrm{G}$ \\
\hline NGC 5273 & Sy 1.9 & 17.8 & $\mathrm{SA} 0^{\circ}(\mathrm{s})$ & 0.84 & $\mathrm{E}$ \\
\hline NGC 5548 & Sy 1.5 & 73.4 & (R')SA0/a(s) & 0.96 & $\mathrm{E}$ \\
\hline \multicolumn{6}{|l|}{ Southern } \\
\hline NGC 1097 & LLAGN & 15.1 & $\mathrm{SB}(\mathrm{s}) \mathrm{b}$ & $\ldots$ & $\mathrm{H}$ \\
\hline NGC 3065 & LLAGN & 28.3 & $\mathrm{SA} 0^{\circ}(\mathrm{r})$ & ... & I \\
\hline IC 4329A & Sy 1.2 & 69.6 & $\mathrm{SA}^{+}$? edge-on & $\ldots$ & $\mathrm{J}$ \\
\hline NGC 2617 & Sy 1.8 & 62.3 & $\mathrm{Sc}$ & $\ldots$ & $\mathrm{K}$ \\
\hline NGC 3783 & Sy1.5 & 44.3 & $\left(\mathrm{R}^{\prime}\right) \mathrm{SB}(\mathrm{r}) \mathrm{ab}$ & $\ldots$ & $\mathrm{L}$ \\
\hline NGC 7213 & LLAGN & 21.1 & SA(s)a? & $\ldots$ & M \\
\hline
\end{tabular}

Note. Column (1): galaxy identification. Column (2): activity type. Column (3): distance (NED, 3K CMD). Column (4): galaxy morphology. Column (5): fractional contribution of broad $\mathrm{H} \alpha$ to the total $\mathrm{H} \alpha+[\mathrm{N} \mathrm{II}]$ emission; an asterisk means that the double-peaked profile was identified only with HST STIS spectra. Column (6): reference for the double-peaked profile: (A) Bower et al. (1996), (B) Denney et al. (2010), (C) Bentz et al. (2006), (D) Shields et al. (2000), (E) Ho et al. (1997d), (F) Ho et al. (2000), (G) Barth et al. (2001), (H) Storchi-Bergmann et al. (1993), (I) Eracleous \& Halpern (2001), (J) Winge et al. (1996), (K) Shappee et al. (2014), (L) Stirpe et al. (1994), (M) Filippenko \& Halpern (1984).

of more distant and luminous type 1 AGN showing similar rms profiles have been reported by Grier et al. (2012a, 2012b).

\section{Type 1 AGN in the Palomar Spectroscopic Survey of Nearby Galaxies}

The Palomar spectroscopic survey (Filippenko \& Sargent 1985; Ho et al. 1995, 1997d)—consisting of spectra of 486 northern galaxies - has a detection limit for emission lines at an equivalent width $\mathrm{EW} \gtrsim 0.25 \AA$. Of the total sample of 486 galaxies, the nuclear spectra of 211 have been classified as AGN, with 46 of them classified as of type 1 .

Table 1 of Ho et al. (1997d) lists the properties of the 46 type 1 nuclei of the Palomar sample: 34 are classed as showing "definite" detection of a broad $\mathrm{H} \alpha$ line, while for the remaining 12 , there is only a "probable" detection of a faint broad $\mathrm{H} \alpha$ component. The fit of the line profiles in these "probable" cases shows that the broad component is not well constrained and may be due instead to complex kinematics of the narrow-line region (NLR). We have inspected the $\mathrm{H} \alpha$ profiles in the Palomar spectra of the 34 nuclei considered to present definite detection of a broad $\mathrm{H} \alpha$ line, and searched in the literature for previous studies with analysis of these profiles. We list in the top part of Table 2 the 12 galaxies that show clearly doublepeaked or flat-topped $\mathrm{H} \alpha$ profiles, giving in the last column of the table the reference in which the profile is shown to be double-peaked (or disk-like). Some of these profiles were found not in the Palomar spectra but in nuclear spectra obtained with the STIS spectrograph aboard the Hubble Space Telescope

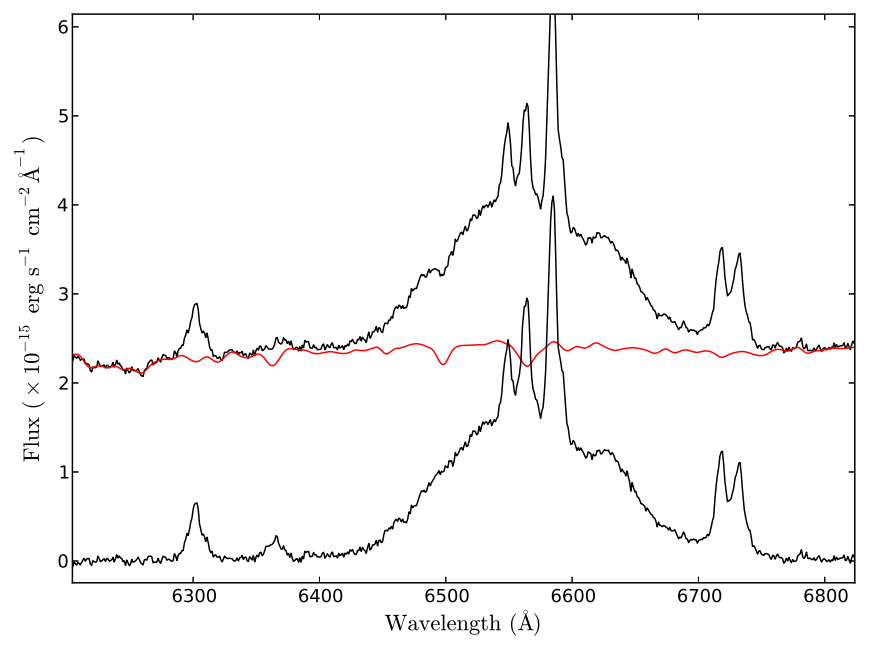

Figure 3. Subtraction of the stellar population template for NGC 4235. Top: Palomar spectrum in black; stellar population template (NGC 4371, from Ho et al. 1997c) in red. Bottom: residual nuclear emission spectrum.

(HST): NGC 4203 (Shields et al. 2000), NGC 4450 (Ho et al. 2000), and NGC 4579 (Barth et al. 2001). The higher angular resolution of the $H S T$ spectroscopy allows the detection of even fainter lines than those detected in the Palomar survey, as the contribution of the stellar population decreases even further in the narrower STIS slits (0"! $1-0$ !" 2$)$.

In order to further support our argument that broad doublepeaked profiles are frequently found in type $1 \mathrm{AGN}$, we list in the bottom part of Table 2 six additional Seyfert 1 galaxies with broad double-peaked or disk-like Balmer line profiles that are not in the Palomar survey (most of them being southern objects), but are type $1 \mathrm{AGN}$ at distances similar to those of the Palomar galaxies. The double-peaked or disk-like profiles of these galaxies were discovered serendipitously.

\subsection{Fit of the Double-peaked Profiles of Type 1 AGN in the Palomar Sample}

In order to further demonstrate that the profiles that appear double-peaked in the Palomar spectra can be attributed to the outer parts of an accretion disk, we now use our model to fit the broad $\mathrm{H} \alpha$ profile in these spectra (Ho et al. 1997d) for the galaxies of Table 2 that showed high enough signal-to-noise ratio in the broad profiles to constrain the fit: NGC 3516, NGC 4151, NGC 4235, NGC 5273, and NGC 5548.

\subsection{Subtraction of the Stellar Component}

Most of the spectra of the above galaxies show strong absorption features, due to the contribution of an underlying stellar population, with the exception of NGC 5548, whose continuum seems to be dominated by the AGN in this spectrum. In order to isolate the gas emission in the other cases, we corrected their nuclear spectra by subtracting a stellar population template, using for this the Palomar spectra of the nonactive galaxies (Ho et al. 1997c) that do not show any emission lines. For each active galaxy, we searched for a stellar population template that reproduced its nuclear spectrum well (in the regions where there are no emission lines) and then subtracted a scaled and Gaussian-convolved version of the chosen template. We used a continuum wavelength window from 6200 to $6250 \AA$ to determine the scale factor between the 

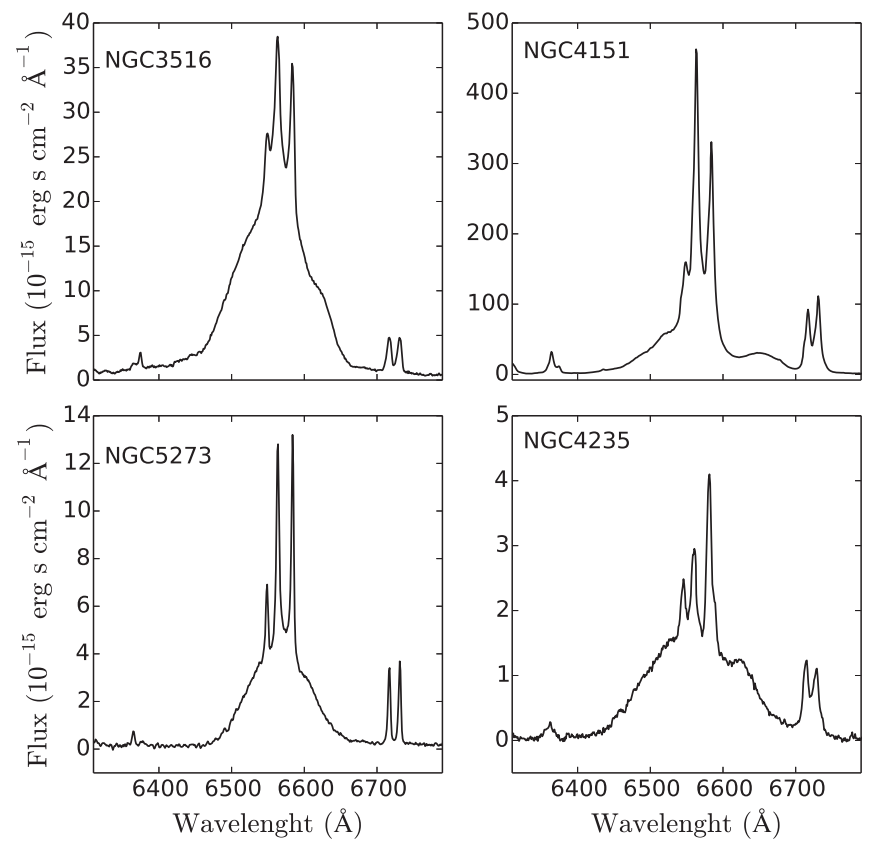

Figure 4. Nuclear emission spectra of NGC 3516, NGC 4115, NGC 4235, and NGC 5273 after the subtraction of the contribution of the stellar population.

nuclear spectrum and the stellar template. This method is very similar to that of Ho et al. (1997c), and the result is illustrated in Figure 3 for NGC 4235. The spectra of the other four galaxies after the subtraction of the stellar component are shown in Figure 4.

\subsection{Fit of the Disk Component}

We have used the "Saturated Spiral Model" (Schimoia et al. 2012) that has provided the best fits in our previous studies of the $\mathrm{H} \alpha$ double-peaked profile of NGC 1097 and in an ongoing study of a series of nuclear spectra of another LINER/Seyfert 1 galaxy, NGC 7213 (J. S. Schimoia et al. 2017, in preparation). We constrained the double-peaked component by requiring it to fit the visible peaks and/or shoulders of the profile, while at the same time trying to fit the wings. In order to reduce the number of free parameters, we fixed the index $q_{2}$ of the outer part of the emissivity law $\left(\xi_{q}<\xi<\xi_{2}\right)$ at $q_{2}=3$, motivated by the fact that for an accretion disk ionized/illuminated by an external source above the disk (as usually adopted as the source of ionization for the gas in the disk) the radial dependence of the surface emissivity is $\epsilon(\xi) \propto \xi^{-3}$ (Dumont \& CollinSouffrin 1990). Only in the case of NGC 4235 did we adopt $q_{2}=2$, as this allowed an improvement in the fit. We also fixed the inner radius where the spiral arm begins at $\xi_{\mathrm{sp}}=\xi_{1}$, which worked well except for NGC4151 (see discussion in Section 4.4).

In order to try to fit the broad wings of the profiles, it was necessary to increase the emissivity of the inner part of the disk (for $\xi_{1}<\xi<\xi_{q}$ ) relative to our previous modeling (e.g., of the double-peaked profile of NGC 1097 and NGC 7213), adopting an index $q_{1}=0$. This index implies a constant emissivity for the region within the radius $\xi_{q}$, while in our previous studies of NGC 1097 and other LINERs the best fit required an emissivity increasing between $\xi_{1}$ and $\xi_{q}$ (with $q_{1}=-2$ ).

\subsubsection{An Additional Broad Component}

J. S. Schimoia et al. (2017, in preparation) monitored the double-peaked profile of the LINER/Seyfert 1 nucleus of NGC 7213 and found that the rms spectrum shows, besides the double-peaked component, a central "hump" in the profile well reproduced by a broad (FWHM $\approx 2200 \mathrm{~km} \mathrm{~s}^{-1}$ ) Gaussian component with centroid velocity close to systemic. We have interpreted this finding as due to the contribution of BLR clouds farther away from the ionizing source than the disk, having thus lower orbital velocity. We find this to be the case also for most Seyfert 1 profiles fitted here: besides the disk component, a broad Gaussian component with centroid velocity close to systemic is also necessary to fit the $\mathrm{H} \alpha$ profile.

In order to constrain this additional component, we proceeded as follows. We fitted the profiles of each of the [S II] $\lambda \lambda 6717,6731$ lines using two Gaussian curves, necessary (and sufficient) in order to fit the profiles, including eventual wings. Each Gaussian fitted to the $\lambda 6731$ line was constrained, in velocity space, to have the same width, relative intensity, and relative central velocity as each corresponding Gaussian fitted to the $\lambda 6717$ line. Then, under the assumption that the narrow components of $\mathrm{H} \alpha$ and [N II] lines originate from the same region as the $[\mathrm{S}$ II] lines, we used the $[\mathrm{S} \mathrm{II}]$ profiles in velocity space to fit the narrow components of the $\mathrm{H} \alpha$ and [N II] $\lambda \lambda 6548,6584$ lines - the last two also constrained to have an intensity ratio of 3 (Osterbrock \& Ferland 2006). The result was that, besides the disk and the constrained narrow components, an additional component-well represented by a broad $\left(1000 \mathrm{~km} \mathrm{~s}^{-1} \leqslant\right.$ FWHM $\left.\leqslant 2000 \mathrm{~km} \mathrm{~s}^{-1}\right)$ Gaussianwas necessary to fit the residual $\mathrm{H} \alpha$ flux in most cases (with the exception of NGC 4273). This procedure is illustrated in Figure 5 for the case of NGC 5273.

The additional broad component was fitted by the following Gaussian curve:

$$
F_{G}(\lambda)=\frac{A_{G}}{\sqrt{2 \pi \sigma_{G}^{2}}} \exp \left[-\frac{\left(\lambda-\lambda_{0}+\Delta \lambda\right)^{2}}{2 \sigma_{G}^{2}}\right],
$$

where $\lambda_{0}$ is the rest wavelength of $\mathrm{H} \alpha, \Delta \lambda$ is the displacement of the Gaussian component relative to the $\mathrm{H} \alpha$ narrow component, $\sigma_{G}$ is the standard deviation, and $A_{G}$ is the amplitude of the Gaussian component.

Following Popović et al. (2004), we have calculated the relative flux contribution between the Gaussian and disk components as

$$
Q=\frac{\int_{-\infty}^{+\infty} F_{G}(\lambda) d \lambda}{\int_{-\infty}^{+\infty} F_{D}(\lambda) d \lambda}
$$

where $F_{D}(\lambda)$ is the flux of the disk component.

\subsection{Results from the Fits}

The parameters derived from the fits of the disk component, as well as the FWHM, velocity shift, and integrated fluxes, are listed in Table 3, where the uncertainties in the parameters were obtained by varying each parameter separately around the bestfit value while keeping all the others parameters fixed. In the bottom part of the table we list the integrated fluxes, FWHM, and velocity shift of the Gaussian component, while in the last line we list the value of the $Q$ parameter. In the last column of Table 3 we list the average values of the disk parameters for all 

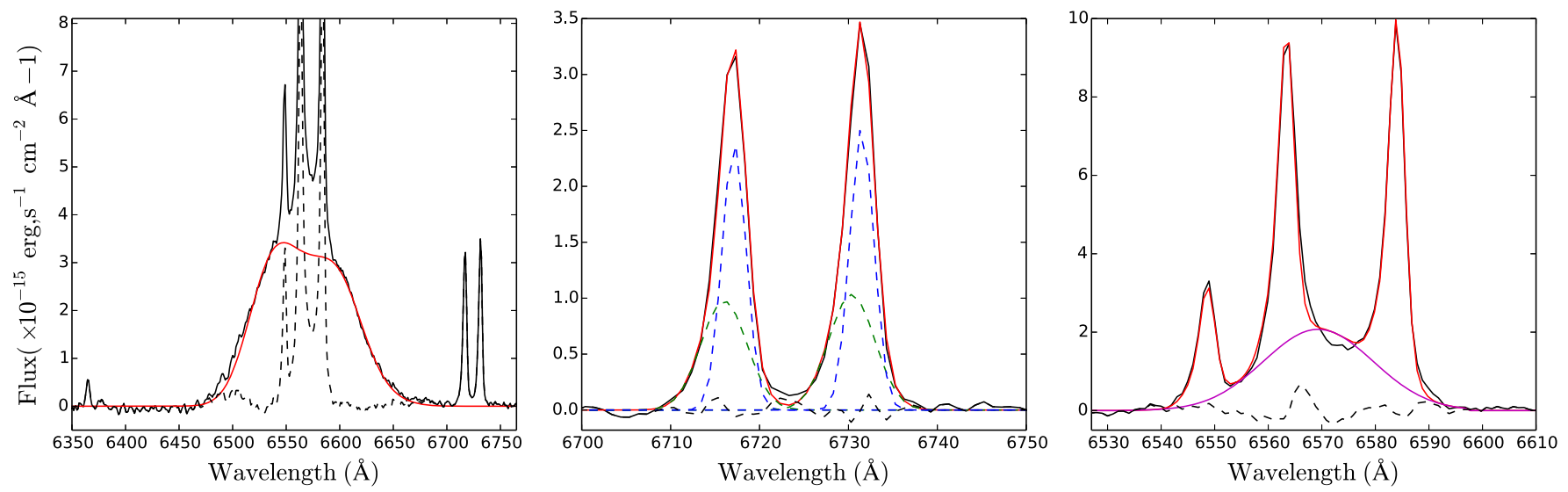

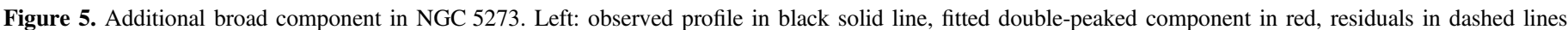

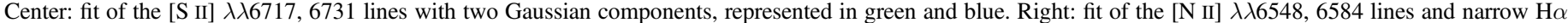

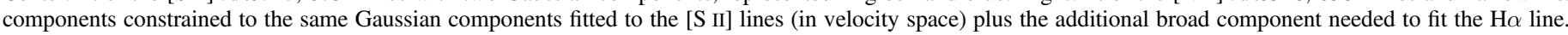

the galaxies and rms deviation from the mean for all the listed properties, including in this average the values for NGC 1097 , listed in Table 1.

Figure 6 shows, in the left column, the fits of the disk and broad Gaussian component to the $\mathrm{H} \alpha$ line, together with those of the narrow components to the $\mathrm{H} \alpha+[\mathrm{N}$ II] and [S II] line profiles. In the central column we show only the disk component overplotted on the emission-line profiles after the subtraction of the broad Gaussian component and narrow components. In all figures, the profiles of the narrow components are well reproduced by the [S II] line profiles, as illustrated for NGC 5273 in Figure 5. The parameters of the disk component, whose profiles are shown in the central column, were used to generate the cartoon shown in the last column of Figure 6, with a rendering of the disk (seen face-on) using the best-fit parameters listed in Table 3.

\subsubsection{The Disk Component}

Table 3, combined with the results of the fit for NGC 1097 in Table 1, shows that the inner radius of the disk ranges between $500 R_{g}$ and $800 R_{g}$, the outer radius between $2500 R_{g}$ and $5500 R_{g}$, and the saturation radius between $1300 R_{g}$ and $2600 R_{g}$. Average values are listed in the last column of Table 3 (we have included in this calculation the values for NGC 1097), showing rms variations of $\sim 30 \%$.

The emissivity distribution of the disk is approximately the same for all Seyfert 1 galaxies: it is constant from the inner radius of the disk up to the saturation radius (emissivity law index $q_{1}=0$ ) and then decreases with emissivity law index of $q_{2}=3$ for all cases but for NGC 4235, whose profile is better fitted with $q_{2}=2$. We note that we have obtained a better fit with $q_{1}=0$ for the Seyfert 1 galaxies than with $q_{1}=-2$ (this worked better for NGC 1097 and NGC 7213). This may indicate a difference in the geometry/structure of the ionizing source between the LLAGN and Seyfert 1 galaxies, such that in the latter the ionizing source shows a larger incidence of ionizing radiation in the inner part of the disk than in the LLAGN, where the incidence or ionizing radiation seems to increase from the inner radius up to the saturation radius.

The spiral arm has a small contrast (between 1 and 2.5) and begins at the inner radius of the disk, with the exception of NGC 4151, in which case we had to make the spiral begin close to the outer border of the disk and give it a much larger contrast
(88) in order to reproduce a strong blue "hump" in the profile. The parameters characterizing the arm in this case are such that the resulting structure can be better described as a "hot spot" in the outer border of the disk.

The inclination angle of the disk (relative to the plane of the sky) is in the range $17^{\circ}<i<38^{\circ}$ (including NGC 1097), meaning that we are seeing the disk closer to face-on than edge-on, in agreement with what is expected for type 1 AGN in the simple unified model (Antonucci 1993).

\subsubsection{The Case of NGC 5548}

The broad $\mathrm{H} \beta$ profiles of NGC 5548 have been modeled by Pancoast et al. (2014b) as originating in a BLR composed of clouds with a flattened distribution between an inner an outer radius, thus in approximate agreement with a disk model like ours. Nevertheless, these authors derive an inclination $i$ for the flat distribution of clouds of $i=39^{\circ} \pm 11^{\circ}$, while we have obtained a much smaller inclination $i=19^{\circ} \pm 2^{\circ}$.

On the other hand, the minimum radius of $1.39 \mathrm{lt}$-day and mean radius of 3.31 lt-day obtained by Pancoast et al. (2014b) correspond to 809 and 1927 gravitational radii $\left(R_{g}\right)$, respectively, for their quoted BH mass of $3.23 \times 10^{7} M_{\odot}$, consistent with our estimate for the inner radius of the line-emitting disk of $\xi_{1}=600 \pm 300\left(R_{g}\right)$ and the saturation radius of $(2600$ $\pm 500) R_{g}$ (see Table 3 ). We also note that, if the mean radius of the disk is a characteristic radius from which most of the line emission originates, as is the case of the saturation radius in our modeling, and we calculate the Keplerian velocity $v_{K}$ at $3.31 \mathrm{lt}-$ day from the $\mathrm{BH}$, we obtain $v_{K}=7086 \mathrm{~km} \mathrm{~s}^{-1}$. If the inclination is $i=19^{\circ}$, the observed velocity should be $v_{K} \sin (i)=2307 \mathrm{~km} \mathrm{~s}^{-1}$, in approximate agreement with our measured FWHM $=5754 \mathrm{~km} \mathrm{~s}^{-1}$ (considering that the velocity is approximately half the FWHM value). For $i=39^{\circ}$, $v_{K} \sin (i)=4459 \quad \mathrm{~km} \mathrm{~s}^{-1}$, which would imply FWHM $\geqslant 9000 \mathrm{~km} \mathrm{~s}^{-1}$, much broader than the value we have measured.

\subsubsection{The Broad Gaussian Component}

The broad Gaussians have FWHM in the range $1000 \mathrm{~km} \mathrm{~s}^{-1} \leqslant \mathrm{FWHM} \leqslant 2000 \mathrm{~km} \mathrm{~s}^{-1}$ and small velocity shifts relative to the centroid velocity of the narrow-line components, in the range $-39 \mathrm{~km} \mathrm{~s}^{-1}>\Delta v>+170 \mathrm{~km} \mathrm{~s}^{-1}$. 
Table 3

Parameters of the Disk plus Broad Gaussian Modeling

\begin{tabular}{|c|c|c|c|c|c|c|}
\hline Component & NGC 3516 & NGC 4151 & NGC 4235 & NGC 5273 & NGC 5548 & $\overline{\text { Average } \pm \mathrm{rms}}$ \\
\hline \multicolumn{7}{|l|}{ Disk } \\
\hline$\xi_{1}$ & $500_{-100}^{+300}$ & $800{ }_{-300}^{+200}$ & $600_{-300}^{+200}$ & $500_{-400}^{+200}$ & $600_{-300}^{+300}$ & $575 \pm 125$ \\
\hline$\xi_{2}$ & $2500_{-300}^{+300}$ & $4000_{-100}^{+100}$ & $5000_{-500}^{+1500}$ & $4000_{-1500}^{+1500}$ & $3500_{-800}^{+800}$ & $3433 \pm 1211$ \\
\hline$\xi_{q}$ & $1500_{-200}^{+400}$ & $1550_{-100}^{+150}$ & $1300_{-300}^{+300}$ & $2000_{-300}^{+400}$ & $2600_{-500}^{+500}$ & $1692 \pm 524$ \\
\hline$i$ & $20_{-2}^{+2}$ & $38_{-2}^{+2}$ & $31_{-3}^{+3}$ & $17_{-2}^{+2}$ & $19_{-2}^{+2}$ & $27 \pm 9$ \\
\hline$\phi_{0}$ & $240_{-30}^{+20}$ & $310_{-5}^{+5}$ & $160_{-5}^{+5}$ & $210_{-30}^{+30}$ & $45_{-25}^{+40}$ & $\ldots$ \\
\hline$q_{1}$ & 0 & 0 & 0 & 0 & 0 & $-0.33 \pm 0.82$ \\
\hline$q_{2}$ & 3 & 3 & 2 & 3 & 3 & $2.8 \pm 0.4$ \\
\hline$A$ & $1.6_{-0.2}^{+0.2}$ & $88_{-5}^{+5}$ & $2.5_{-0.5}^{+1.5}$ & $1.0_{-0.4}^{+0.4}$ & $1.5_{-0.2}^{+0.2}$ & $\ldots$ \\
\hline$p$ & $-20_{-30}^{+5}$ & $90_{-60}^{+60}$ & $-30_{-4}^{+6}$ & $90_{-50}^{+50}$ & $-20_{-50}^{+15}$ & $27 \pm 57$ \\
\hline$\delta\left(^{\circ}\right)$ & $90_{-20}^{+40}$ & $20_{-2}^{+2}$ & $60_{-30}^{+30}$ & $30_{-20}^{+20}$ & $30_{-10}^{+10}$ & $50 \pm 28$ \\
\hline$\sigma$ & $750{ }_{-50}^{+50}$ & $500_{-50}^{+50}$ & $500_{-100}^{+100}$ & $850_{-100}^{+150}$ & $900_{-50}^{+50}$ & $733 \pm 189$ \\
\hline FWHM $\left(\mathrm{km} \mathrm{s}^{-1}\right.$ & $6932_{-680}^{+680}$ & $8959_{-1052}^{+685}$ & $7986_{-272}^{+454}$ & $5374_{-273}^{+228}$ & $5754_{-101}^{+134}$ & $7001 \pm 1501$ \\
\hline Shift $\left(\mathrm{km} \mathrm{s}^{-1}\right)$ & 0 & 457 & 229 & 0 & -457 & $38 \pm 303$ \\
\hline Flux & 1434 & 5636 & 174 & 234 & 3783 & $2252 \pm 2390$ \\
\hline \multicolumn{7}{|l|}{ Broad Gaussian } \\
\hline FWHM $\left(\mathrm{km} \mathrm{s}^{-1}\right)$ & 1919 & 1981 & $\ldots$ & 1101 & 885 & $1472 \pm 560$ \\
\hline Shift $\left(\mathrm{km} \mathrm{s}^{-1}\right)$ & 68 & 171 & $\ldots$ & 149 & -39 & $87 \pm 95$ \\
\hline Flux & 678 & 3785 & $\ldots$ & 53 & 416 & $1233 \pm 1720$ \\
\hline$Q$ & 0.47 & 0.67 & $\ldots$ & 0.23 & 0.11 & $0.37 \pm 0.25$ \\
\hline
\end{tabular}

Note. Units of $\xi_{1}, \xi_{2}$, and $\xi_{q}$ are gravitational radii. Fluxes units are $10^{-15} \mathrm{erg} \mathrm{cm}^{-2} \mathrm{~s}^{-1}$. The orientation of the spiral arm $\phi_{0}$ is measured counterclockwise from the bottom of Figure 6 at the inner radius where it begins (with the exception of NGC 4151). Uncertainties in fluxes and FWHM values are smaller than 10\%. The calculated average values (plus the standard deviation rms) listed in column (7) include the fitting parameters for NGC 1097 listed in Table 1.

We attribute these components to more distant clouds from the SMBH than the disk, thus having lower velocities.

The parameter $Q$ that measures the contribution of the Gaussian component relative to the disk component ranges between 0.11 and 0.67 , with the Gaussian being strongest for NGC $4151(Q=0.67)$ and NGC $3516(Q=0.47)$.

The presence of double-peaked disk components in nearby type 1 AGN is also supported by RM studies of the $\mathrm{H} \beta$ broadline profile. Denney et al. (2010) find that five of the six Seyfert 1 nuclei they studied (which include NGC 3516 and NGC 5548) show double-peaked or flat-topped broad $\mathrm{H} \beta$ profiles in either the mean or rms spectrum (or both). The same is found for five other more distant and luminous type 1 AGN by Grier et al. (2012a, 2012b). Peterson et al. (2004) show that the rms spectrum of NGC 4151 is also doublepeaked, while Doroshenko et al. (2012) show the same also for Mrk 6.

\section{The Statistics of Double-Peaked Profile Emitters Among Type 1 Nuclei}

Type 1 AGN in the Palomar Survey constitute between 34 (with "unambiguous" broad lines) and 46 (including broad lines that could be due to multiple components in the narrow lines) of the 211 AGN, thus $\sim 20 \%$ (Ho et al. 1997d; Ho 2008). We will use this percentage to put approximate constraints on the half-opening angle $\theta_{m}$ of the obscuring torus in the simple approximation of the unified model scenario, in which the geometry of the escaping radiation is that of a cone (Antonucci 1993). We now know that the "torus" is most likely a distribution of dusty clouds, and that its properties vary with the AGN luminosity (e.g., Elitzur 2012; Ichikawa et al. 2015), but the simple unified model can give us some insight on relative probabilities. In the simple model, the $20 \%$ fraction of type 1 AGN implies that the "free" solid angle of the AGN is $2 \pi\left[1-\cos \left(\theta_{m}\right)\right] \approx 20 \%$ of $2 \pi$, with $80 \%$ of $2 \pi$ being the solid angle covered by the obscuring torus. Thus, in order to see directly the AGN-the accretion disk and BLR-our line of sight has to make an angle of at most $\theta_{m} \approx 37^{\circ}$ relative to the AGN symmetry axis.

In order to illustrate how the double-peaked profiles would appear as a function of the orientation of our line of sight relative to the AGN, we show in Figure 7 a series of doublepeaked profile models for inclinations ranging from $0^{\circ}$ to $35^{\circ}$. The parameters of the model are the average values in Table 3 we have obtained from the fitted models to the Palomar spectra applied to the $\mathrm{H} \alpha$ profile, which is the most prominent line in the optical spectra of AGN (as it is $\geqslant 3$ times as strong as $\mathrm{H} \beta$ ). In this figure, we have superposed the narrow components of $\mathrm{H} \alpha$ and [N II] $\lambda \lambda 6548,6584$ on the broad double-peaked profile. These lines were simulated by Lorentzians with $\mathrm{FWHM}=300 \mathrm{~km} \mathrm{~s}^{-1}$.

Figure 7 shows that, in order for $\mathrm{H} \alpha$ to appear doublepeaked, our line of sight has to make an angle of at least $\sim 20^{\circ}$ relative to the ionization axis of $\mathrm{AGN}$. This means that the disk inclination should be between $\sim 20^{\circ}$ and $\sim 37^{\circ}$ for the profile to be observed as double-peaked. The inclination angles derived from our fits are between $17^{\circ}$ and $38^{\circ}$, thus in good agreement with the predicted range. Previous fits of similar accretion disk models to double-peaked profiles, such as those observed in radio galaxies (Chen \& Halpern 1989; Eracleous \& Halpern 1994; Lewis et al. 2010) and nearby LLAGN (Ho et al. 2000; Storchi-Bergmann et al. 2003), also show a similar range of inclinations. Considering the inclination values listed in Table 3 for the five targets we have fitted with our disk model 

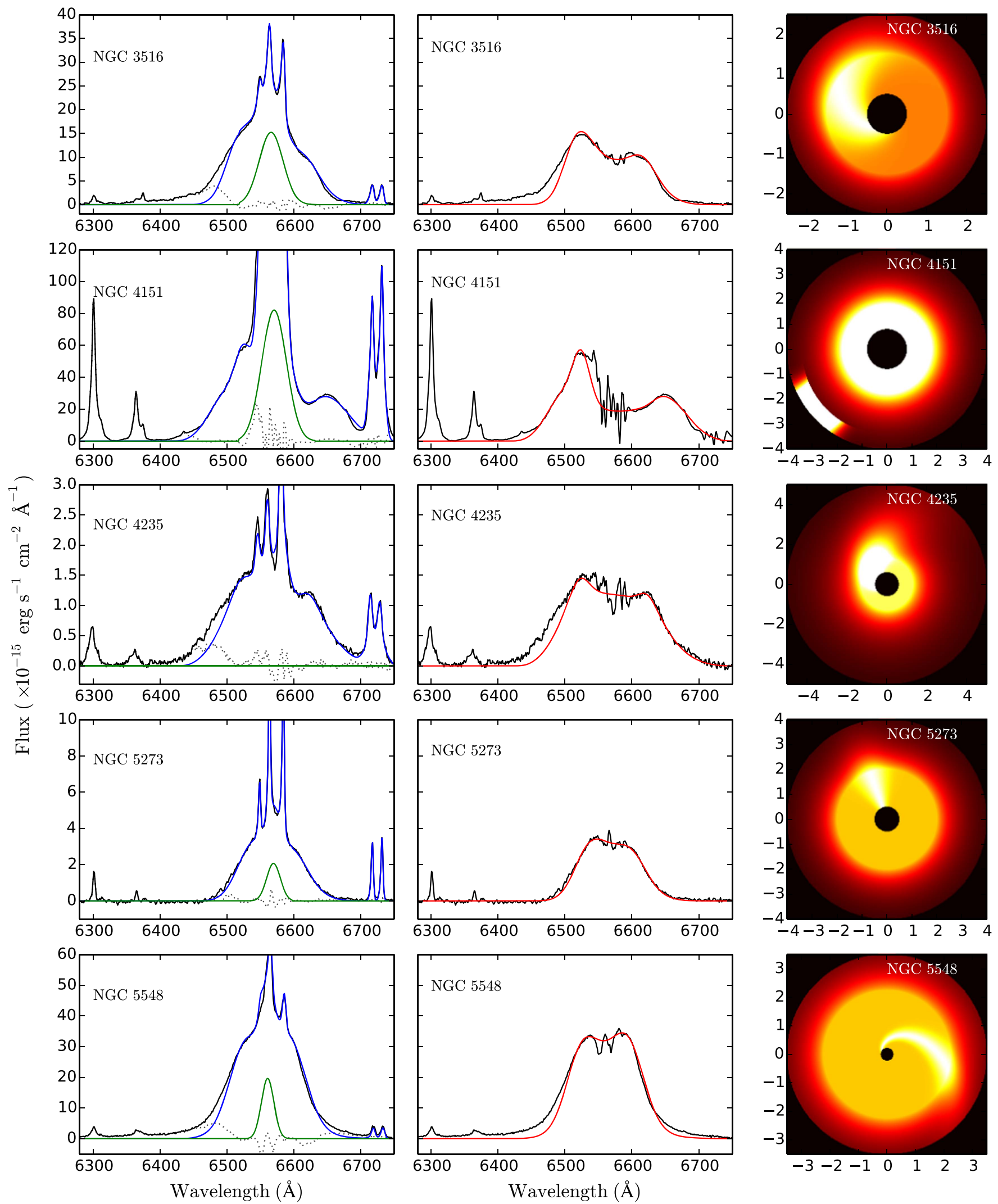

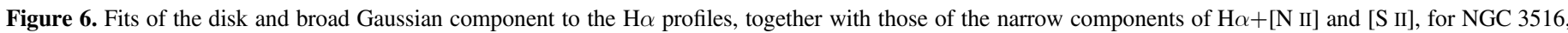

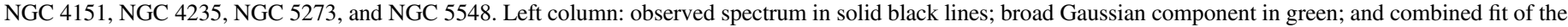

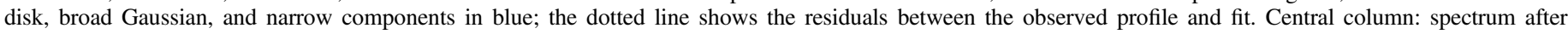

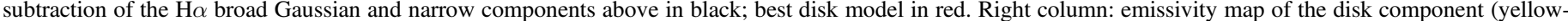

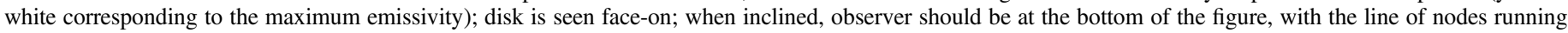
along the horizontal direction. Axis labels are in units of $1000 \xi$. 

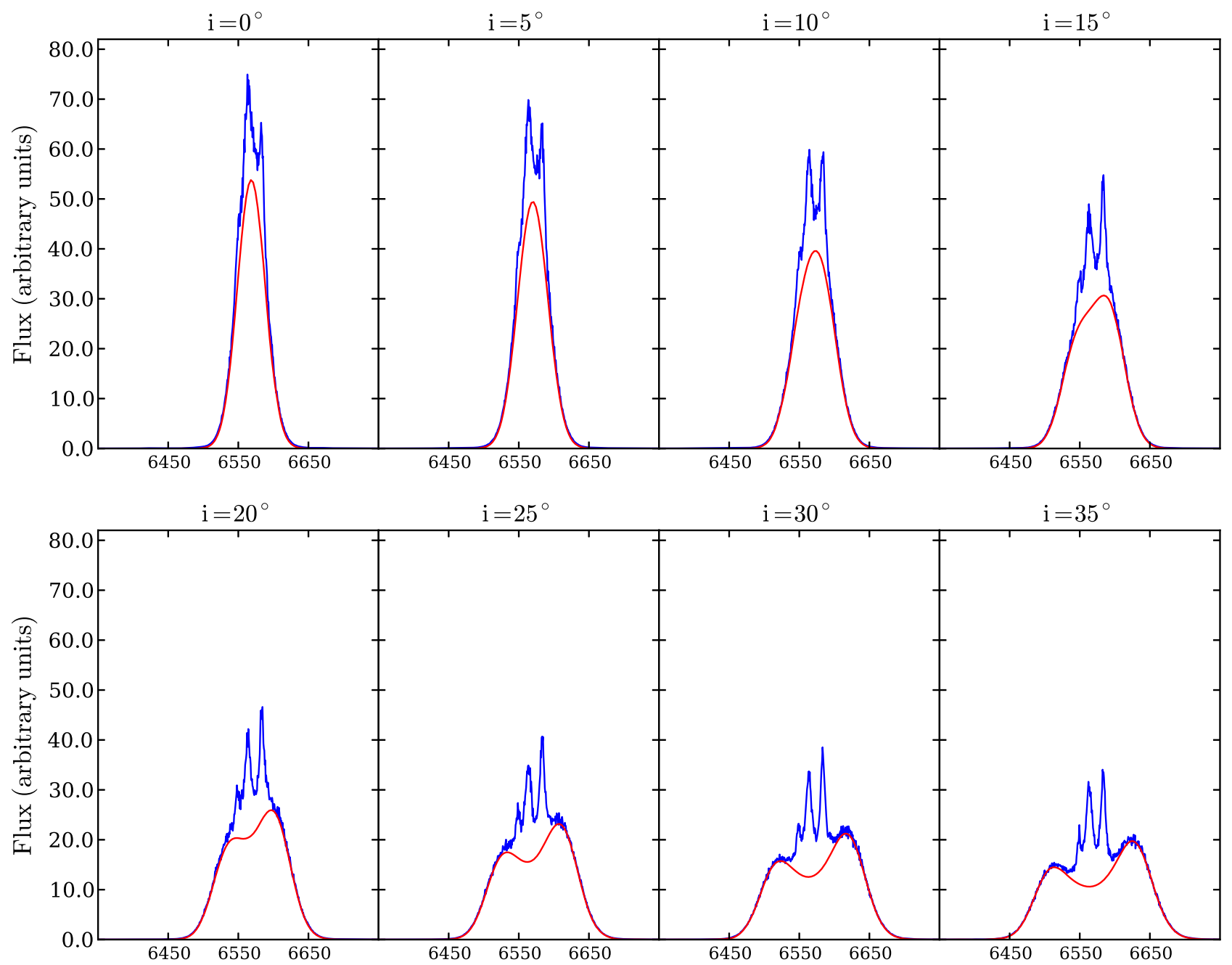

Figure 7. Disk model for the $\mathrm{H} \alpha$ profile as a function of inclination of the disk relative to the plane of the sky. Typical narrow $\mathrm{H} \alpha+[\mathrm{N}$ II] emission-line profiles have been superimposed on the broad double-peaked profiles.

plus the fit to NGC 1097, we obtain a mean value for the inclination of the disk of $i=27^{\circ} \pm 9^{\circ}$.

Assuming again the conical geometry of the simple model, the solid angle for inclinations between $20^{\circ}$ and $35^{\circ}$ is $12 \%$ of $2 \pi$. When we compare this number with the $20 \%$ fraction above for type $1 \mathrm{AGN}$, we conclude that $\sim 60 \%$ of type 1 AGN should have double-peaked Balmer line profiles. According to these predictions, if the total sample of type 1 nuclei in the Palomar sample is 34 galaxies, we should expect to find $\sim 20$ galaxies with double-peaked profiles. We find 12, thus $\sim 35 \%$ instead of the predicted $60 \%$. On the other hand, the number we have found may be a lower limit, as, in some cases, the doublepeaked nature of the line profiles could be observed only in very high quality $H S T$ STIS spectra in which the contrast between the emission lines and the host-galaxy starlight is much higher than in the Palomar spectra. In addition, in the small aperture of the STIS spectra, the NLR contribution can also be minimized, increasing the contrast between the BLR and NLR emission. As not all the type 1 AGN of the Palomar sample have been observed with HST STIS, this fraction of $35 \%$ double-peakers may be a lower limit.

Another consideration is that, in the Palomar spectra, a critical property of the broad line to allow its clear detection is its flux compared with the narrow lines. Inspection of the fits in Figures 4-14 of Ho et al. (1997d) shows that clear broad $\mathrm{H} \alpha$ components can only be detected when the fraction of the flux in the broad line is larger than 50\% of the total flux in the broad plus narrow $\mathrm{H} \alpha+[\mathrm{N}$ II] lines. This fraction, obtained from Ho et al. (1997d), is listed in the fifth column of Table 2. The total number of these sources from Ho et al. (1997d) is 14, with 9 (64\%) of them having double-peaked profiles, now consistent with the $\sim 60 \%$ prediction above. Another possibility is that the ratio of the outer to inner radius of the disk is $\gg 10$, which would make the line profiles singly peaked (see Figure 9 of Eracleous \& Halpern 2003). Finally, if the accretion rate is high, we would expect a contribution from nondisk clouds in the form of a single broad Gaussian component, as discussed in the previous section. This component would "fill" the doublepeaked "dip" in the profile, making it difficult to distinguish the double-peaked component in the total emission-line profile.

\section{Discussion}

As mentioned in Section 1, the fact that broad-line profiles of Seyfert 1 and radio galaxies seem to contain a flattened component has been noted in previous studies, including also those by Wills \& Browne (1986), Chen \& Halpern (1989), Jackson \& Browne (1991), Rokaki et al. (1992), Brotherton (1996), Marziani et al. (1996), and Wanders \& Peterson (1996). More recently, Pancoast et al. (2014a, 2014b) have performed 
detailed dynamical modeling of the BLR of five Seyfert 1 nuclei. The main conclusion of Pancoast et al. (2014b) is that the BLR geometry is a thick disk viewed close to face-on, in agreement with our findings. They argue also for the presence of inflows that could be due to the motion of BLR clouds in elliptical orbits around the SMBH (Elitzur et al. 2014). Another possibility is that the Gaussian component represents perturbations in the surface brightness of the disk that are not captured by the single spiral arm we have adopted.

On the basis of this and previous studies, we suggest that the disk is present in most BLRs, and at the higher Eddington ratios of Seyfert 1 galaxies, we observe additional line-emitting components corresponding to nondisk clouds, usually at lower velocities (Ghayuri 2016). Some of these clouds may be inflowing (Grier et al. 2013b), may be just orbiting the SMBH in elliptical orbits as proposed by Pancoast et al. (2014b), or may be part of an outflowing disk wind (Elitzur et al. 2014).

\subsection{Implications for the Derivation of Black Hole Masses}

The technique of RM has provided valuable tools for the estimation of the mass of SMBHs in AGN up to very large distances in the universe, solely on the basis of the observed continuum luminosity and width of the broad emission lines. The optical luminosity allows the estimate of the BLR radius (Wandel et al. 1999; Kaspi et al. 2005; Bentz et al. 2013), while the width of the broad profile provides the velocity dispersion of the BLR clouds $\Delta V$. Assuming that the motion of the BLR gas is dominated by gravity and that radiation pressure can be neglected, the mass of the central black hole is given by (Peterson et al. 2004)

$$
M_{\mathrm{BH}}=f \frac{R_{\mathrm{BLR}} \Delta V^{2}}{G},
$$

where $R_{\mathrm{BLR}}$ is the radius of the BLR, $G$ is the gravitational constant, and $f$ is a dimensionless numerical factor that depends on the structure, kinematics, and orientation of the BLR. A recent empirical determination of the average scale factor is $\langle f\rangle=4.31 \pm 1.05$ (Grier et al. 2013a), based on the assumption that the relationship between black hole mass and bulge velocity dispersion is the same for quiescent and active galaxies. The value of $f$ is of fundamental importance in using Equation (7) for the determination of the masses of SMBHs across the universe and incorporates the uncertainties due to the geometry and inclination of the BLR.

The recognition that the disk component may be ubiquitous in type $1 \mathrm{AGN}$ has important implications for the value of $f$ in the cases dominated by the disk component. If we consider that the geometry of the region that varies on the shortest timescale probed by the profile variation is a flat disk, $f=1 / \sin ^{2} i$, where $i$ is the inclination of the disk relative to the plane of the sky, as the observed width of the lines $\Delta V$ is simply the rotational speed projected into the line of sight. In particular, for $\langle f\rangle=4.3,\langle i\rangle=29^{\circ}$, which is practically the same as the median value that we obtain from the data in Table 3 , of $i=27^{\circ} \pm 9^{\circ}$.

Similar disk inclinations have also been reported from the fit of disk models to double-peaked profiles in previous studies (Chen \& Halpern 1989; Eracleous \& Halpern 1994, 2003).

The fact that the majority of disks are observed with inclinations between $20^{\circ}$ and $37^{\circ}$ is supported also by predictions of the simple model: if we consider that the

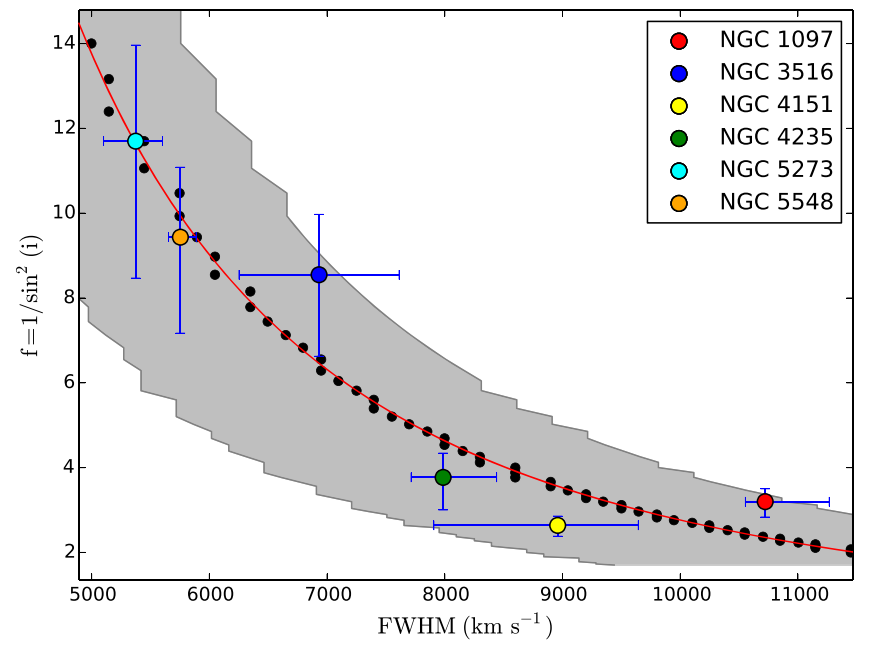

Figure 8. Relation between $f=1 / \sin ^{2} i$ and the FWHM $\left(\mathrm{km} \mathrm{s}^{-1}\right)$ of the broad line according to our average model (see last column of Table 3), represented by black dots for inclinations in the range $10^{\circ} \leqslant i \leqslant 50^{\circ}$, and for the broad profiles of the type 1 AGN of Table 3 plus NGC 1097 from Table 1 . The gray band shows the range of the model values corresponding to the rms variations of the parameter values listed in the last column of Table 3 . The red line is a fit of the relation implied by the models.

maximum opening angle of the torus is $\theta_{m} \approx 37^{\circ}$, then the fraction of type $1 \mathrm{AGN}$ observed with inclinations between $20^{\circ}$ and $37^{\circ}$ should be $\sim 80 \%$, while only $\sim 20 \%$ should be observed with inclinations smaller than $20^{\circ}$. This seems to be the case of NGC 5273, for which we obtain $i=17^{\circ}$, and of NGC 3227, for which we fit the rms profile and obtain an inclination of $i=16^{\circ}$. These low inclinations imply a larger value, $f \sim 12$. Using this $f$ value in Equation (7) would result in a mass for the SMBH 3 times larger than that obtained using the average $f$ value. Intriguingly (or coincidentally), the SMBH mass of NGC 3227 obtained using RM is a factor of 2-3 lower than the dynamical mass estimates for this galaxy: Denney et al. (2010) give $M_{\mathrm{RM}}=6.0 \times 10^{6} M_{\odot}$ (using $f=4.3$ ), while Hicks \& Malkan (2008) give $2.0 \times 10^{7} M_{\odot}$ from gas kinematics, and Davies et al. (2006) —using stellar kinematics -provide a $1 \sigma$ range spanning these two, i.e., $6.0 \times 10^{6}-2.0 \times 10^{7} M_{\odot}$.

\subsection{A Relation between $\mathrm{f}$ and the FWHM of the Broad Lines}

According to our model-which applies for the case in which the BLR is dominated by emission from the disk-the adoption of an ensemble average value for $f$ is a good approximation for $80 \%$ of type 1 AGN (even though there should be some variation between targets), but for the $20 \%$ cases of more pole-on AGN the use of the constant $f$ implies an underestimation of the SMBH mass.

Our model implies $f=1 / \sin ^{2} i$ for the disk component. The facts that (1) the parameters of the disk, and in particular their inner, outer, and saturation radii, do not vary much among different type 1 AGN ( 30\%; see Table 3) and (2) the width (FWHM) of the line strongly depends on the inclination of the disk (see Figure 7) imply that there should be a relation between the FWHM of the broad profiles and $f$.

We have used our model to obtain this relation between $f$ and FWHM of the profiles as follows. We have fixed the disk parameter values at the average values listed in the last column of Table 3, except for the inclination $i$. We have called this model the "average model." We have then varied the 
inclination $i$ between $10^{\circ}$ and $50^{\circ}$, to build a sequence of models as a function of $i$. For each model we measured the value of the FWHM (in $\mathrm{km} \mathrm{s}^{-1}$ ) and plotted $f$ as a function of FWHM in Figure 8 as black circles. We have then fitted the relation between these two quantities with the function

$$
f=\frac{(5.1 \pm 0.3) \times 10^{9}}{\text { FWHM }^{(2.30 \pm 0.04)}} .
$$

This relation is shown as a red line in Figure 8. We have also included in Figure 8 the values obtained from the fit of the profiles of the five galaxies in Table 3 plus that of NGC 1097 in Table 1, shown as colored circles. The error bars correspond to the uncertainties in the inclinations listed in Table 3 and in the FWHM values. The latter uncertainties were obtained as follows. While the listed FWHM values were obtained considering as maximum flux the average between the peak fluxes of red and blue peaks or shoulders, the maximum FWHM value was adopted as corresponding to a maximum flux equal to the flux of the lower peak and the minimum FWHM value as corresponding to a maximum flux equal to the flux of the higher peak. Figure 8 shows that the measured FWHM values follow the relation given by Equation (8) within the uncertainties.

Lower values of FWHM correspond to low disk inclinations, for which it is important to consider the thickness of the disk. For a ratio between a half thickness $H$ and disk radius $R, f$ becomes (Collin et al. 2006)

$$
f=\frac{1}{(H / R)^{2}+\sin ^{2} i} .
$$

Although this expression ensures that the value of $f$ will not diverge for low inclinations, we have checked that, for a typical value of $H / R=0.1$ (Collin et al. 2006), the decrease in the value of $f$ by including the term $H / R$ is at most $\sim 10 \%$ for the range of inclinations we are considering $\left(i \geqslant 16^{\circ}\right)$.

The gray band in Figure 8 constitutes the range of the model values corresponding to the rms variations of the parameter values listed in the last column of Table 3 . The variations of the regression parameters of Equation (8) allow the coverage of the gray band in Figure 8.

Equation (8) implies that, approximately, $f \propto \mathrm{FWHM}^{-2}$. We note that Equation (7) is also consistent with this relation if the ratio $M_{\mathrm{BH}} / R_{\mathrm{BLR}}$ is constant. Our modeling, in which the profile shape depends on the inner, outer, and maximum emissivity radii in units of gravitational radii, implies exactly this: that the profile shape depends only on the ratio between the disk radii and the SMBH mass and that this ratio seems to be approximately constant.

The fact that $f$ does not depend much on the ratio $M_{\mathrm{BH}} / R_{\mathrm{BLR}}$ is supported also by the following argument. From Equation (7) we have

$$
f \propto \frac{M_{\mathrm{BH}}}{R_{\mathrm{BLR}}} \frac{1}{\Delta V^{2}},
$$

and we also know that $R_{\mathrm{BLR}} \propto L^{1 / 2}$ and that $L \propto \dot{M}$. The Eddington ratio is defined as $\dot{m}=\dot{M} / \dot{M}_{\text {Edd }}$ and $\dot{M}_{\text {Edd }} \propto M_{\mathrm{BH}}$. So $\dot{M} \propto \dot{m} \dot{M}_{\mathrm{Edd}} \propto \dot{m} M_{\mathrm{BH}}$. Therefore,

$$
\frac{M_{\mathrm{BH}}}{R_{\mathrm{BLR}}} \propto \frac{M_{\mathrm{BH}}}{L^{1 / 2}} \propto \frac{M_{\mathrm{BH}}}{\left(\dot{m} M_{\mathrm{BH}}\right)^{1 / 2}}
$$

and

$$
f \propto\left(\frac{M_{\mathrm{BH}}}{\dot{m}}\right)^{1 / 2} \frac{1}{\Delta V^{2}} .
$$

Thus, even for varying SMBH masses and accretion rates, the dependence of $f$ on these properties is weak, and, as a result, $f$ depends mostly on the width of the profile: $f \propto \Delta V^{-2}$ or $f \propto \mathrm{FWHM}^{-2}$.

\subsection{Further Implications for the Structure of AGN}

It has been argued that the broad double-peaked Balmer lines in LLAGN and radio galaxies originate in the outer parts of an accretion disk that is ionized by an ion torus (Chen \& Halpern 1989) or other radiatively inefficient structure (Narayan 2005; Nemmen et al. 2006). Such structure develops when the mass accretion rate to the SMBH drops below some minimum Eddington ratio, of order $\sim 1 \%$, which is the case for LLAGN.

LLAGN and Seyfert 1 nuclei have been compared to the two states observed in X-ray binaries (Remillard \& McClintock 2006; Narayan \& McClintock 2008): the low-luminosity hard state and the more luminous soft state (or thermal state), respectively. The thermal state is the one dominated by the geometrically thin, optically thick disk, analogous to more luminous AGN (Seyfert galaxies and quasars), where the UV/ optical emission is dominated by the "big blue bump" (BBB) from the accretion disk. The low-luminosity hard state is characterized by a harder X-ray spectrum and much lower thermal emission from the thin disk, and this is consistent with the spectral energy distribution of LLAGN, which lacks the BBB component.

The fact that the double-peaked profiles are observed both in LLAGN and in Seyfert 1 galaxies suggests that the inner structures of the low-luminosity and high-luminosity AGN are not very different. The disk component seems to be always present. In LLAGN, the disk emission is the only component seen, while in more luminous type 1 sources, such as Seyfert 1 nuclei and quasars, there is an additional broad-line component that, since the velocity dispersion is smaller, probably originates in more distant clouds than the gas that produces the disk component. This is also supported by RM studies- the fact the double-peaked characteristic pops out in the rms spectrum, which shows only variable emission on RM timescales, suggests that the lower-velocity gas "filling in" the peak is either optically thin to the continuum or else at a larger distance, where geometrical damping of the shorttimescale RM variations occurs (much like the NLR).

The observation of the nondisk clouds leading to the additional broad component can be understood as due to the higher luminosity of the source; at higher accretion rates there should be more gas clouds in the vicinity of the nucleus, either inflowing or originating from an accretion disk wind that gets enhanced with increased accretion rates.

\section{Conclusions}

We have used previously published results, as well as fits of broad $\mathrm{H} \alpha$ profiles in the spectra of nearby active type 1 nuclei from the Palomar Survey of Nearby Galaxies, to argue that disk-like emission-line profiles-double-peaked profiles originating in the outer parts of an accretion disk, at $\sim 1000$ Schwarzschild radii-are ubiquitous in the BLR of these objects. 
We have shown that while "pure" disk profiles are seen mostly in LLAGN, in Seyfert 1 galaxies the profiles usually present an additional narrower component (FWHM $\sim 1000-2000 \mathrm{~km} \mathrm{~s}^{-1}$ ) well fitted with a Gaussian function. We attribute this component to nondisk BLR gas, usually moving at lower velocities. Our results support previous studies arguing that, while for LLAGN the accretion rate is low and most accreting gas is confined to the disk, for the higher accretion rates that occur in Seyfert galaxies, one can also observe lower-velocity ionized gas that is orbiting the SMBH farther from the disk, in inflow toward the disk or being ejected in a wind from the disk.

The disk component-being closer to the ionizing sourceshould be the most variable part of the profile, and indeed many RM studies show that the rms spectrum reveals a doublepeaked profile.

While previous studies have already argued that the BLR is dominated by a flattened component, our study — via the fit of a disk component to the BLR profiles-has allowed us to obtain the parameters of the disk, such as the inner and outer radii and the maximum emissivity radius in units of gravitational radii. We have found that these parameters do not vary by more than $30 \%$ among the different sources, which leads to the conclusion that the main parameter that regulates the width of the doublepeaked profile is the inclination of the disk relative to the plane of the sky.

In the cases for which the BLR emission is dominated by the disk component, the factor $f$ in the formula used to calculate the SMBH mass $M_{\mathrm{BH}}=f\left(R_{\mathrm{BLR}} \Delta V^{2} / G\right)$ should be $f=1 / \sin ^{2} i$. The median inclination of the sources fitted with the disk model in our study, $i=27^{\circ}$, corresponds to $f=4.5$, very close to the most recent value of $\langle f\rangle=4.3 \pm 1.05$ (Grier et al. 2013a), obtained in a completely independent way. But the range of inclinations we obtained, $17^{\circ} \leqslant i \leqslant 38^{\circ}$, implies that the $f$ factors range between $\sim 2.6$ and $\sim 12$. Thus, for the lowest inclinations (narrower profiles), the SMBH masses obtained using a fixed value of $f$ are underestimated by a factor of $\approx 3$.

Having concluded that the main parameter regulating the width of the BLR profiles - in the disk-dominated cases-is the inclination of the disk, our study has further allowed us to propose a relation (Equation (8)) between the value of $f$ and the FWHM of the broad profiles that could lead to an improvement in the determination of SMBH masses in disk-dominated AGN.

We thank the referee for a careful reading of the manuscript and valuable suggestions that helped to improve the paper. T.S. B. acknowledges support from Conselho Nacional de Desenvolvimento Científico e Tecnológico $(\mathrm{CNPq})$. B.M.P. is grateful for support by the NSF through grant AST-1008882 to The Ohio State University. K.D.D. is supported by an NSF AAPF fellowship awarded under NSF grant AST-1302093. R. N. acknowledges financial support from Fundação de Amparo à Pesquisa do Estado de São Paulo (FAPESP).

\section{References}

Antonucci, R. 1993, ARA\&A, 31, 473

Barth, A., Ho, L. C., Filippenko, A. V., Rix, H.-W., \& Sargent, W. L. W. 2001, ApJ, 546, 205

Bentz, M. C., Denney, K. D., Cackett, E. M., et al. 2006, ApJ, 651, 775

Bentz, M. C., Denney, K. D., Cackett, E. M., et al. 2007, ApJ, 662, 205

Bentz, M. C., Denney, K. D., Grier, C. J., et al. 2013, ApJ, 767, 149

Bentz, M. C., Walsh, J. L., Barth, A. J., et al. 2009, ApJ, 705, 199

Bentz, M. C., Walsh, J. L., Barth, A. J., et al. 2010, ApJ, 716, 993
Bon, E., Popović, L. C., avrilović, N., Mura, G. L., \& Mediavilla, E. 2009, MNRAS, 400, 924

Bower, G. A., Wilson, A. S., Heckman, T. M., \& Richstone, D. O. 1996, AJ, 111,1901

Brotherton, M. S. 1996, ApJS, 102, 1

Chen, K., \& Halpern, J. P. 1989, ApJ, 344, 115

Chen, K., Halpern, J. P., \& Filippenko, A. V. 1989, ApJ, 339, 742

Collin, S., Kawaguchi, T., Peterson, B. M., \& Vestergaard, M. 2006, A\&A, 456, 75

Davies, R. I., Thomas, J., Genzel, R., et al. 2006, ApJ, 646, 754

Denney, K. D., Assef, R. J., Horne, K., Peterson, B. M., \& Vestergaard, M. 2012, ASPC, 460, 103

Denney, K. D., Peterson, B. M., Pogge, R. W., et al. 2010, ApJ, 721, 715

Dietrich, M., Peterson, B. M., Albrecht, P., et al. 1998, ApJS, 115, 185

Dietrich, M., Peterson, B. M., Grier, C. J., et al. 2012, ApJ, 757, 53

Doroshenko, V. T., Sergeev, S. G., Klimanov, S. A., Pronik, V. I., \& Efimov, Y. S. 2012, MNRAS, 426, 416

Dumont, A. M., \& Collin-Souffrin, S. 1990, A\&A, 229, 313

Elitzur, M. 2012, ApJL, 744, L33

Elitzur, M., Ho, L. C., \& Trump, J. R. 2014, MNRAS, 438, 3340

Eracleous, M., \& Halpern, J. P. 1994, ApJS, 90, 1

Eracleous, M., \& Halpern, J. P. 2001, ApJ, 554, 240

Eracleous, M., \& Halpern, J. P. 2003, ApJ, 599, 886

Filippenko, A. V., \& Halpern, J. P. 1984, ApJ, 285, 458

Filippenko, A. V., \& Sargent, W. L. W. 1985, ApJS, 57, 503

Gaskell, C. M. 1983, in Proc. 24th Liège Int. Ap. Coll., ub Quasars and Gravitational Lenses (Liège: Institut d'Astrophysique, Université Liège), 473

Gezari, S., Halpern, J. P., \& Eracleous, M. 2007, ApJS, 169, 167

Ghayuri, M. 2016, MNRAS, 462, 490

Gilbert, A. M., Eracleous, M., Filippenko, A. V., \& Halpern, J. P. 1999, in ASP Conf. Ser. 175, ed. C. M. Gaskell et al. (San Francisco, CA: ASP), 189

Goad, M., \& Wanders, I. 1996, ApJ, 469, 113

Grier, C. J., Martini, P., Watson, L. C., et al. 2013a, ApJ, 773, 90

Grier, C. J., Peterson, B. M., Horne, K., et al. 2013b, ApJ, 764, 47

Grier, C. J., Peterson, B. M., Pogge, R. W., et al. 2012a, ApJ, 755, 60

Grier, C. J., Peterson, B. M., Pogge, R. W., et al. 2012b, ApJL, 744, L4

Hicks, E. K. S., \& Malkan, M. A. 2008, ApJS, 174, 31

Ho, L. C. 2008, ARA\&A, 46, 475

Ho, L. C., Filippenko, A. V., \& Sargent, W. L. W. 1995, ApJS, 98, 477

Ho, L. C., Filippenko, A. V., \& Sargent, W. L. W. 1997, ApJS, 112, 315

Ho, L. C., Filippenko, A. V., Sargent, W. L. W., \& Peng, C. Y. 1997, ApJS, 112,391

Ho, L. C., Rudnick, G., Rix, H. W., et al. 2000, ApJ, 541, 120

Ichikawa, K., Packham, C., Ramos Almeida, C., et al. 2015, arXiv:1501.06584 Jackson, N., \& Browne, I. W. A. 1991, MNRAS, 250, 422

Jovanovic, P., Popovic, L. C., Stalevski, M., \& Shapovalova, A. I. 2010, ApJ, 718,168

Kaspi, S., Maoz, D., Netzer, H., et al. 1996, ApJ, 470, 336

Kaspi, S., Maoz, D., Netzer, H., et al. 2005, ApJ, 629, 61

La Mura, Di Mille, F., Ciroi, S., Popović, L. Ć., \& Rafanelli, P. 2009, ApJ, 693, 1437

Lewis, K. T., Eracleous, M., \& Storchi-Bergmann, T. 2010, ApJS, 187, 416

Maoz, D., Netzer, H., Mazeh, T., et al. 1991, ApJ, 367, 493

Marziani, P., Sulentic, J. W., Dultzin-Hacyan, D., Calvani, M., \& Moles, M. 1996, ApJS, 104, 37

Narayan, R. 2005, Ap\&SS, 300, 177

Narayan, R., \& McClintock, J. E. 2008, NewAR, 51, 733

Nemmen, R. S., Storchi-Bergmann, T., Yuan, F., et al. 2006, ApJ, 643, 652

Netzer, H., Maoz, D., Laor, A., et al. 1990, ApJ, 353, 108

Osterbrock, D. E., \& Ferland, G. J. 2006, Astrophysics of Gaseous Nebulae and Active Galactic Nuclei (2nd ed.; Mill Valley, CA: Univ. Science Books)

Pancoast, A., Brewer, B. J., Treu, T., et al. 2014a, MNRAS, 445, 3055

Pancoast, A., Brewer, B. J., Treu, T., et al. 2014b, MNRAS, 445, 3073

Peterson, B. M., Berlind, P., Bertram, R., et al. 2002, ApJ, 581, 197

Peterson, B. M., Ferrarese, K., Gilbert, K. M., et al. 2004, ApJ, 613, 682

Peterson, B. M., Korista, K. T., \& Cota, S. A. 1987, ApJL, 312, L1

Popović, L. Č., Mediavilla, E., Bon, E., \& Ilić, D. 2004, A\&A, 423, 909

Popovic, L. C., Shapovalova, A. I., Illić, D., et al. 2011, A\&A, 528, 130

Remillard, R. A., \& McClintock, J. E. 2006, ARA\&A, 44, 49

Rokaki, E., Boisson, C., \& Collin-Souffrin, S. 1992, A\&A, 253, 57

Schimoia, J. S., Storchi-Bergmann, T., Grupe, D., et al. 2015, ApJ, 800, 63

Schimoia, J. S., Storchi-Bergmann, T., Nemmen, R. S., Winge, C., \& Eracleous, M. 2012, ApJ, 748, 145

Sergeev, S. G., Doroshenko, V. T., Dzyuba, S. A., et al. 2007, ApJ, 668, 708 
Sergeev, S. G., Klimanov, S. A., Doroshenko, V. T., et al. 2011, MNRAS, 410, 1877

Sergeev, S. G., Pronik, V. I., Peterson, B. M., Sergeeva, E. A., \& Zheng, W. 2002, ApJ, 576, 660

Sergeev, S. G., Pronik, V. I., \& Sergeeva, E. A. 2000, A\&A, 356, 41

Shapovalova, A. I., Popović, L. C.., Burenkov, A. N., et al. 2010, A\&A, 517, A42

Shapovalova, A. I., Popović, L. Č., Burenkov, A. N., et al. 2013, A\&A, 559, A10

Shappee, B. J., Prieto, J. L., Grupe, D., et al. 2014, ApJ, 788, 48

Shields, J. C., Rix, H.-W., McIntosh, D. H., et al. 2000, ApJL, 534, L27

Stirpe, G., Winge, C., Altieri, B., et al. 1994, ApJ, 425, 609

Storchi-Bergmann, T., Baldwin, J. A., \& Wilson, A. S. 1993, ApJL, 410, L11
Storchi-Bergmann, T., Nemmen da Silva, R., Eracleous, M., et al. 2003, ApJ, 598, 956

Strateva, I., Strauss, M. A., Hao, L., et al. 2003, AJ, 126, 1720

Veilleux, S., \& Zheng, W. 1991, ApJ, 377, 89

Wandel, A., Peterson, B. M., \& Malkan, M. A. 1999, ApJ, 526, 579

Wanders, I., \& Peterson, B. M. 1996, ApJ, 466, 174

Wanders, I., Goad, M. R., Korista, K. T., et al. 1995, ApJL, 453, L87

Wills, B. J., \& Browne, I. W. A. 1986, ApJ, 302, 56

Winge, C., Peterson, B. M., Horne, K., et al. 1995, ApJ, 445, 680

Winge, C., Peterson, B. M., Pastoriza, M. G., \& Storchi-Bergmann, T. 1996, ApJ, 469, 648

Zheng, W., Sulentic, J. W., \& Binette, L. 1990, ApJ, 365, 105 